\title{
Modified High-Sucrose Diet-Induced Abdominally Obese and Normal-Weight Rats Developed High Plasma Free Fatty Acid and Insulin Resistance
}

\author{
Li Cao, ${ }^{1}$ Xuehui Liu, ${ }^{2}$ Hongyi Cao, ${ }^{3}$ Qingguo $\mathbf{~},{ }^{2}$ and Nanwei Tong ${ }^{2}$ \\ ${ }^{1}$ Division of Geriatrics, West China Hospital of Sichuan University, Chengdu 610041, China \\ ${ }^{2}$ Division of Endocrinology and Metabolism, West China Hospital of Sichuan University, Chengdu 610041, China \\ ${ }^{3}$ Division of Endocrinology, The Fifth People's Hospital of Chengdu, Chengdu 611130, China
}

Correspondence should be addressed to Nanwei Tong, buddyjun@hotmail.com

Received 29 June 2012; Revised 31 October 2012; Accepted 26 November 2012

Academic Editor: Peter Adhihetty

Copyright () 2012 Li Cao et al. This is an open access article distributed under the Creative Commons Attribution License, which permits unrestricted use, distribution, and reproduction in any medium, provided the original work is properly cited.

\begin{abstract}
Introduction. Metabolically obese but normal-weight (MONW) individuals have metabolic features of overt obesity, and abdominal adiposity is common in them. Animal models of MONW individuals are lacking. We aimed to develop an abdominally obese and normal-weight (AONW) rat model. Methods and Results. Young male Sprague-Dawley rats were fed chow or a modified high-sucrose (HS) diet for 20 weeks. The HS diet induced increased visceral adipose tissue without increased body weight, reduced glucose disposal rates, and increased hepatic glucose output during the hyperinsulinemic-euglycemic clamp, increased plasma glucose during the intraperitoneal glucose tolerance test, and increased plasma free fatty acids. Hepatic lipidosis and hepatocyte mitochondria swelling were found in HS rats through light microscopy and transmission electron microscopy; similar impairments were not observed in muscle. RT-PCR showed that mRNA expression of uncoupling protein 3 and peroxisome proliferatoractivated receptor-gamma coactivator $1 \alpha$ increased in muscle of HS rats, while expression of mitochondrial transcription factor A, glucose transporter type 4, and insulin receptor substrate-1 did not change significantly. Conclusion. AONW rats developed metabolic disorders seen in MONW individuals. Steatosis, mitochondrial morphologic changes, and insulin resistance were more serious in liver than in muscle. Genes involved in fatty acid metabolism and mitochondrial function changed in less impaired muscle.
\end{abstract}

\section{Introduction}

There is a special group of individuals who are in normalweight but have metabolic features observed in overt obesity. These individuals are not obese on the basis of height and weight, but are hyperinsulinemic, insulin-resistant, and predisposed to type 2 diabetes, hypertriglyceridemia, and premature coronary heart disease. They have been identified as metabolically obese but normal-weight (MONW) individuals [1]. MONW individuals are very prevalent in the general population [2-6]. Although they have a normal body mass index or are mildly obese, MONW individuals usually have obviously increased visceral adipose as measured on dual X-ray absorptiometry, computed tomography, and magnetic resonance imaging $[3,7,8]$. The increased visceral fat is strongly associated with insulin resistance and other metabolic disorders in MONW individuals [8].

An animal model of abdominal obesity without obviously increased body weight would be valuable to study metabolic disorders mimicking the situations in MONW individuals. However, almost all available rat models with metabolic disturbances [9-11] are obese models with insulin resistance.

High-fat and high-sucrose diets are the most frequently used diets in the establishment of rat models of insulin resistance. Compared with high-fat diets, high-sucrose diets do not increase the body weight of rats in the long term [1214]. Thus, we attempted to develop a rat model of abdominal obesity with normal weight (AONW) using a modified high-sucrose diet that provided a smaller proportion of 
calories from sucrose than that in general high-sucrose formulas. The metabolic characteristics, insulin sensitivity, and morphological changes in liver and muscle were measured. Gene expressions involved in mitochondrial function and metabolism of fatty acids and glucose in muscle were detected in this rat model.

\section{Materials and Methods}

2.1. Animals and Diets. 4- to 5-week-old male SpragueDawley rats obtained from the Huaxi Animal Centre of Sichuan University were randomly assigned to either the control group (CG) or the HS diet group (HS). The total calories contained in $100 \mathrm{~g}$ of each diet were 349 and $365 \mathrm{kcal}$ in the CG and HS, respectively. Carbohydrate, protein, and fats provided $62 \%, 17 \%$, and $21 \%$ of calories, respectively, in the CG, and $70 \%, 20 \%$, and $10 \%$ of calories, respectively, in the HS. In the HS diet, sucrose and starch provided 35\% of calories. The chow was offered by the university animal centre. The amount of daily diet was not limited in either group. Rats were housed in cages with 12 hours of light (7:00-19:00), a temperature of $21^{\circ} \mathrm{C}$ to $26^{\circ} \mathrm{C}$, and $60 \%$ to $70 \%$ humidity. Rats were weighed every 4 days during the first 4 weeks, then weekly from 5 to 20 weeks.

2.2. Intraperitoneal Glucose Tolerance Test. After 19 weeks of treatment, rats were fasted for 12 hours before the intraperitoneal glucose tolerance test (IPGTT). A 10\%-glucose solution $(2 \mathrm{~g} / \mathrm{kg})$ was intraperitoneally injected in awake and fasted rats. Blood samples were sequentially collected via the tail vein before and 30,60, and 120 min after injection.

\subsection{Hyperinsulinemic-Euglycemic Clamp and Metabolic Mea-} surement. After 20 weeks of treatment, rats were studied from 8:00 after fasting overnight. After anesthesia by intraperitoneal injection of pentobarbital, cannulae were inserted into the right jugular vein and left carotid artery for blood sampling and infusions, respectively. After successful cannula insertion, a blood sample was collected for measurement of lipid profiles. When rats began to recover from anesthesia in 1 hour, insulin was infused at a rate of $4 \mathrm{U} \cdot \mathrm{kg}^{-1} \cdot \mathrm{min}^{-1}$ while euglycemia was maintained by infusion of $20 \%$ glucose. At $60 \mathrm{~min}$ before infusion of insulin and glucose, an initial sample was collected for measurement of basic insulin and glucose. At the same time, a bolus of $\left[{ }^{3} \mathrm{H}\right]$ glucose $(6 \mu \mathrm{Ci})$ in $0.5 \mathrm{~mL}$ of saline was injected, followed by infusion at $0.1 \mu \mathrm{Ci} / \mathrm{min}$ until the end of the experiment. After the infusion of insulin and unlabeled glucose, a blood sample was taken every $10 \mathrm{~min}$ to measure the blood glucose concentration for adjustment of the glucose infusion rate (GIR) until blood glucose levels reached a steady state (basic glucose $\pm 0.1 \mathrm{mmol} / \mathrm{L}$ ), then every $5 \mathrm{~min}$ until the end of the experiment. Blood samples $(0.5 \mathrm{~mL})$ were taken at 10,30 , $60,90,100,110$, and $120 \mathrm{~min}$. The glucose disappearance rate $\left(R_{d}\right)$ and hepatic glucose output rate were calculated from the disappearance of $\left[{ }^{3} \mathrm{H}\right]$ glucose. Plasma levels of insulin during the clamp were obtained from averaged values of blood samples taken in the steady state. Insulin was detected by radioimmunoassay (Linco, USA). Serum triglyceride and cholesterol were examined by an enzymatic method (Dongou, China). Plasma free fatty acids (FFA) were examined by chromatometry (Randox, UK).

2.4. Histopathology. After recovering from the above-described operation, rats were sacrificed in the fasted state with an overdose of intraperitoneal pentobarbital at 9:00. Liver, gastrocnemius, and vastus lateralis were rapidly removed and fixed for histologic section. The slices were stained by hematoxylin/eosin and Sudan III and observed under a microscope at 150x.

2.5. Electron Microscopy. For transmission electron microscopy (TEM), muscles were fixed in $2.5 \%$ glutaraldehyde for 24 hours. Photos were captured using an H-600IV transmission electron microscope (Hitachi Ltd., Japan) in the TEM laboratory of Sichuan University.

2.6. Real-Time Fluorescent Quantitative PCR. The rats' total RNA from $30 \mathrm{mg}$ of gastrocnemius muscle was isolated with Trizol reagent (Gibco, USA). The total RNA was dissolved in $100 \mu \mathrm{L}$ of DEPC-treated water and stored at $-80^{\circ} \mathrm{C}$ until further use. RNA integrity was assessed by electrophoresis in $1 \%(\mathrm{wt} / \mathrm{vol})$ agarose minigels and ethidium bromide staining. cDNA was synthesized according to the instructions of RevertAid First Strand cDNA Synthesis Kit (MBI, Lithuania). TaqMan primers and probes were offered by Shanghai Biotech Company according to the array of uncoupled protein 3 (UCP3), peroxisome proliferator-activated receptor-gamma coactivator $1 \alpha$ (PGC- $1 \alpha)$, mitochondrial transcription factor A (TFAM), glucose transporter type 4 (GLUT4), and insulin receptor substrate-1 (IRS-1) in NCBI GenBank using Primer Express Software (PE Applied Biosystems, CA). The RT-qPCR reaction was carried out in an FTC 2000 Real-Time PCR System (Fenglyn Company, Canada).

2.7. Statistical Analysis. All values are presented in figures and tables as sample means \pm SE. Statistical analysis was performed using a two-tailed paired Student's $t$-test or ANOVA for CG versus HS to establish effects of the intervention. The type I error rate was set a priori at $P<0.05$. Analysis was performed using SPSS 13.0.

\section{Results}

3.1. High-Sucrose Diet Induced Abdominally Obese Rats with Normal Weight. As shown in Figure 1, weight gains did not differ between the groups during the first 20 weeks of the study period $(P=0.636)$. After 20 weeks, weight gain in the HS increased more rapidly than in the CG (data not shown). However, as shown in Figure 2, the mesenteric, retroperitoneal, epididymal, and total intraperitoneal fat weights in the HS increased obviously compared with those in the CG $(P=0.061,0.021,0.008$, and 0.012 , resp.). Among these, the epididymal fat increased most significantly. Thus, the HS rats could be called AONW rats. 


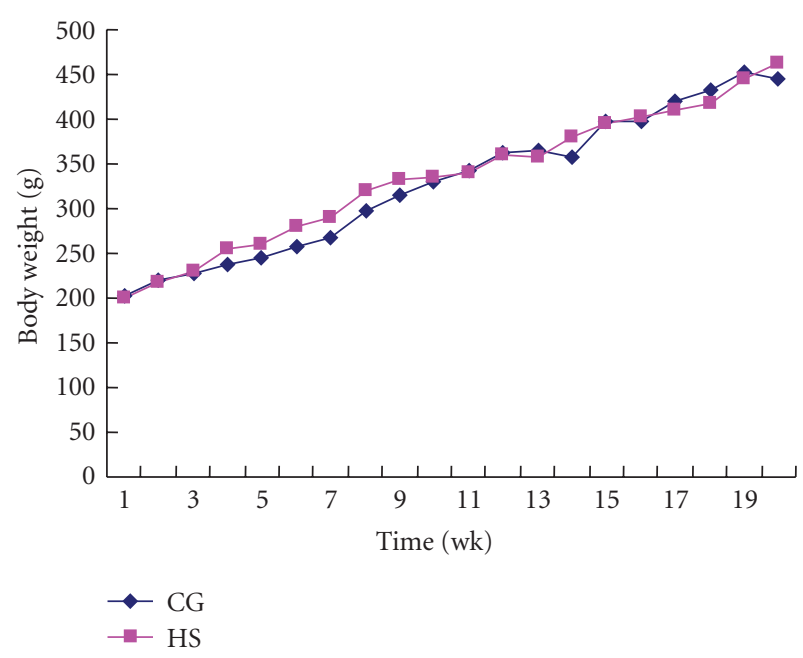

Figure 1: Body weights of rats in control group (CG) and highsucrose diet group (HS) during the first 20 weeks of the study. Values are means $\pm \mathrm{SD}, n=9, P=0.636$.

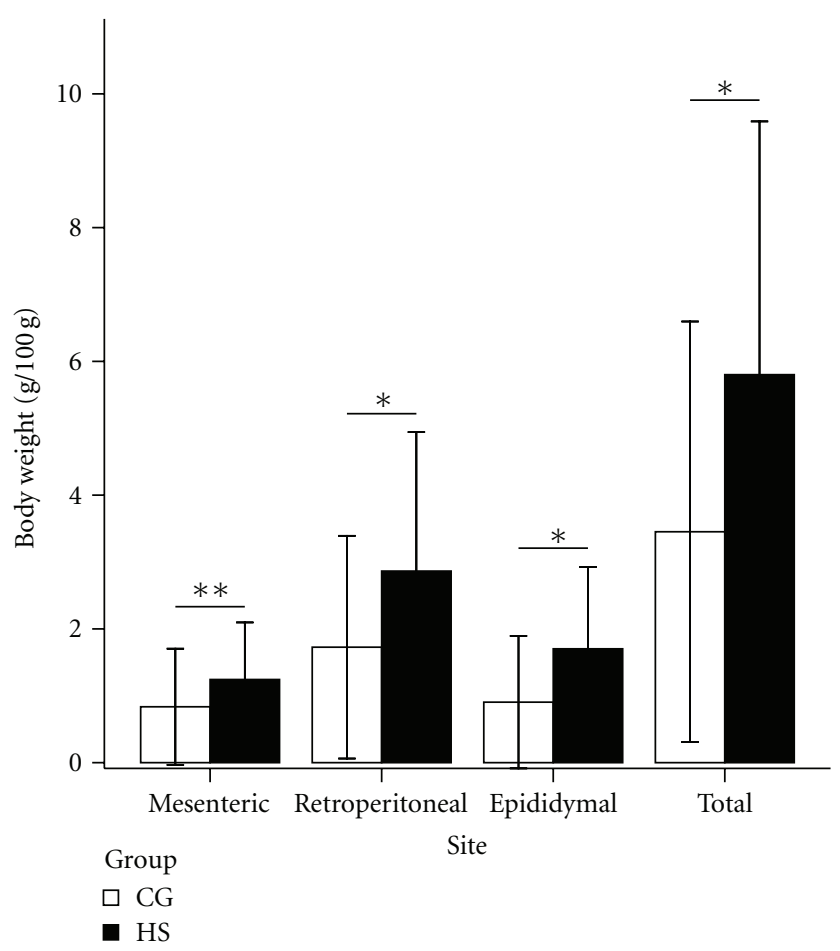

FIgURe 2: Visceral fat weight per $100 \mathrm{~g}$ body weight of rats in the control group (CG) and high-sucrose diet group (HS). The error bar is \pm 2 SD. $n=9$. ${ }^{*} P<0.05,{ }^{* *} P=0.06$. BW: body weight.

3.2. AONW Rat Features Increased Plasma FFA and Glucose Intolerance. As shown in Figure 3, the serum triglyceride, total cholesterol, plasma glucose, and plasma insulin levels in the fasting state showed no differences between the two groups, while the fasting plasma FFA level increased $(P<$ $0.05)$ in AONW rats. In the IPGTT, AONW rats showed increased serum glucose levels at 30,60, and $120 \mathrm{~min}$ (Figure 4). The area under the blood glucose curve in the
IPGTT was $31.83 \pm 5.59$ in AONW rats and $25.71 \pm 4.67$ in controls $(P=0.023)$. AONW rats showed glucose intolerance.

3.3. AONW Rats Showed Insulin Resistance in Liver and Peripheral Tissue. Data from the hyperinsulinemic-euglycemic clamp studies after 20 weeks are shown in Table 1. In the basal state, no differences in the glucose disposal rate (GDR) or hepatic glucose output (HGO) were found between the two groups. In the stable state during the clamp, the HS diet induced a significant decrease in GIR compared with the CG diet $(P<0.01)$. The GDR of peripheral tissues was also significantly reduced in the HS $(P<0.05)$ during the clamp. There was a trend that the HGO could not be inhibited by insulin in the HS, as in the CG. The inhibited rate of HGO by insulin in AONW rats was obviously reduced compared with that in the CG, although the difference was not significant because of the large SD. The inhibited rate of HGO was only $38 \%$ of that in CG rats.

3.4. Hepatic Lipidosis and Mitochondrial Changes in Liver of AONW Rats. The liver of AONW rats showed lipidosis under Sudan III stain (Figure 5). Under electronic microscope, AONW rats showed mitochondrial swelling and mitochondrial cristae were decreased. More glycogenosomes were observed in the AONW rats (Figure 6).

3.5. Muscle Morphology of AONW Rat Hind Legs. In rat hind legs, the morphology of mitochondria did not differ between the CG and HS although the content of mitochondria was not measured. Lipid droplets were found in the HS muscle. Muscle fibers of the HS presented capillary necrosis (Figure 7).

3.6. Expression of Genes Involved in Fatty Acid Metabolism and Mitochondrial Function in Muscle of AONW Rat Hind Legs. Because the insulin-resistant muscle did not show obvious morphologic changes, we explored whether expression of some important genes involved in FFA metabolism, glucose uptake, and mitochondrial function had been modulated. The results of RT-PCR are shown in Figure 8. The UCP3 and PGC- $1 \alpha$ mRNA expression levels increased significantly in AONW rats. TFAM, GLUT4, and IRS-1 mRNA expression levels were not statistically changed.

\section{Discussion}

This study showed that a diet containing 35\% of caloric intake from sucrose led to insulin resistance in rats, while body weights did not increase during feeding for 20 weeks. As expected, the insulin-resistant rats with normal weights developed highly increased VAT. Thus, this model could be called an AONW rat model. The AONW rats developed high plasma FFA, impaired glucose tolerance as demonstrated by the IPGTT, and insulin resistance as shown by the hyperinsulinemic-euglycemic clamp, which are metabolic features of MONW individuals $[4,8]$. Thus, the characteristics of AONW rats mimic situations in MONW individuals to some 


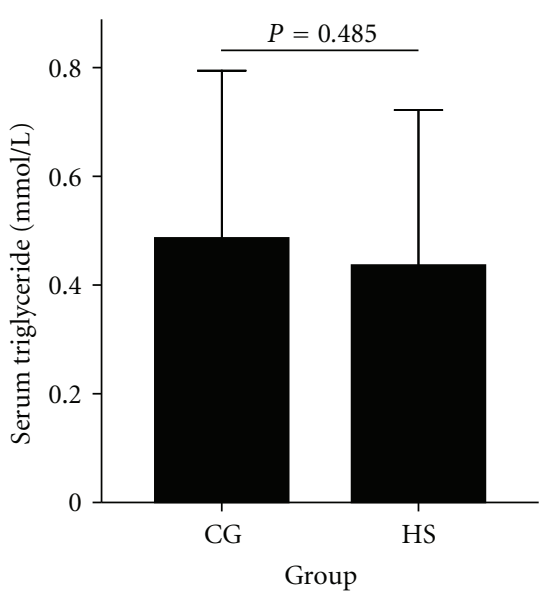

(a)

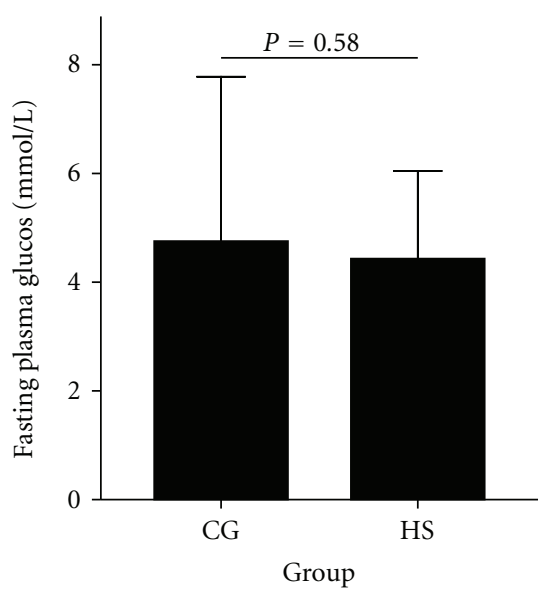

(d)

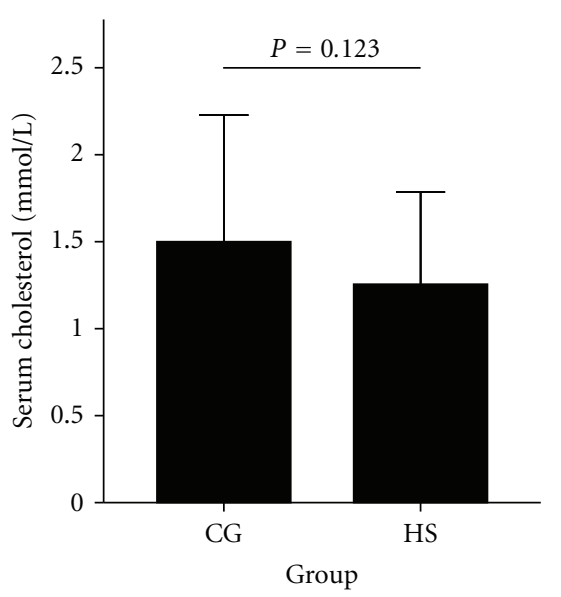

(b)

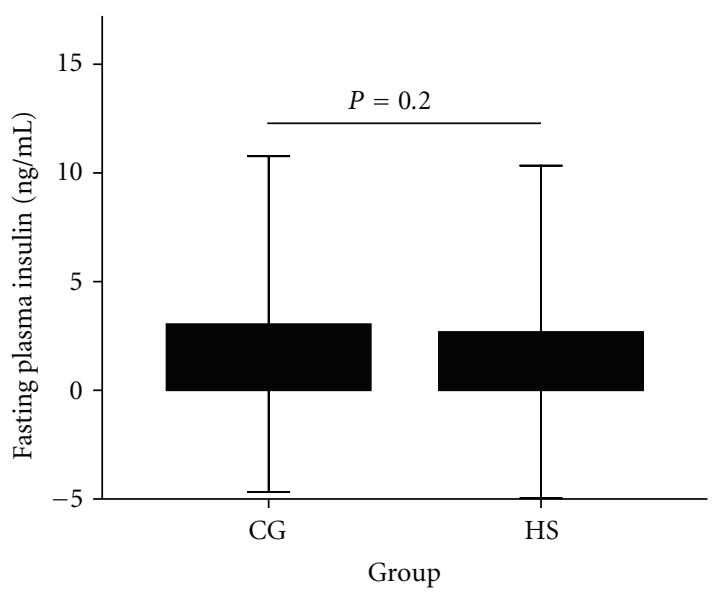

(e)

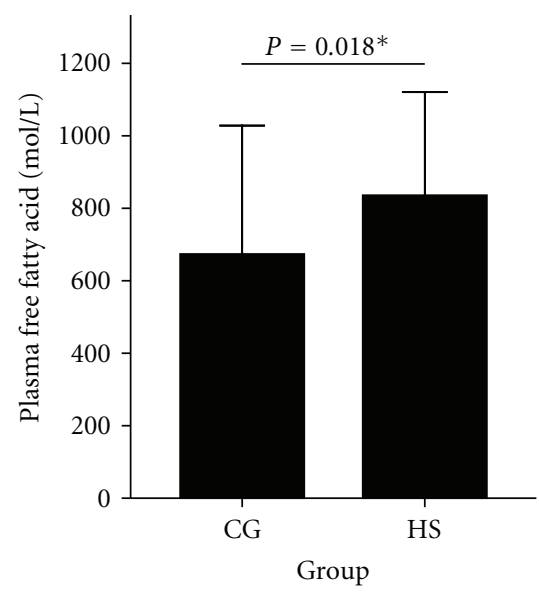

(c)

FiguRe 3: Fasting serum triglycerides (a), total cholesterol (b), plasma free fatty acids (c), plasma glucose (d), and plasma insulin (e) at 20 weeks. The error bar is \pm 2 SD. CG: control group. HS: high-sucrose group.

TABLE 1: Parameters of glucose metabolism from hyperinsulinemic-euglycemic clamp.

\begin{tabular}{|c|c|c|c|c|}
\hline & CG $(n=9)$ & HS $(n=9)$ & $t$ values & $P$ \\
\hline Basal GDR $\left(\mathrm{mg} \cdot \mathrm{min}^{-1} \cdot \mathrm{kg}^{-1}\right)$ & $7.98 \pm 2.59$ & $8.48 \pm 3.50$ & -0.35 & 0.74 \\
\hline Clamp GDR $\left(\mathrm{mg} \cdot \mathrm{min}^{-1} \cdot \mathrm{kg}^{-1}\right)$ & $16.57 \pm 5.41$ & $10.75 \pm 5.16$ & 2.34 & 0.03 \\
\hline Clamp GIR $\left(\mathrm{mg} \cdot \mathrm{min}^{-1} \cdot \mathrm{kg}^{-1}\right)$ & $12.80 \pm 4.41$ & $3.47 \pm 2.03$ & 5.72 & 0.00 \\
\hline Clamp HGO $\left(\mathrm{mg} \cdot \mathrm{min}^{-1} \cdot \mathrm{kg}^{-1}\right)$ & $3.77 \pm 4.52$ & $7.28 \pm 6.01$ & -1.40 & 0.18 \\
\hline Inhibited rate of $\mathrm{HGO}^{\S}$ & $0.60 \pm 0.49$ & $0.23 \pm 0.57$ & 1.45 & 0.17 \\
\hline
\end{tabular}

Values are means $\pm \mathrm{SD}$

GDR: glucose disposal rate by peripheral tissues, mainly skeletal muscles; GIR: glucose infusion rate; HGO: hepatic glucose output. § Inhibited rate of HGO is defined as (Clamp HGO - Basal HGO)/Basal HGO.

CG: control group; HS: high-sucrose group.

extent and could be used to study abdominal adiposityrelated metabolic disorders. This is valuable because biopsy cannot be performed in humans in many situations.

In the hyperinsulinemic-euglycemic clamp, the AONW rats showed significantly reduced GIR, which demonstrated a decreased overall capability of disposing of ectogenic glucose. GDR in AONW rats was reduced to $64.2 \%$ of that in CG rats, which demonstrated insulin resistance of peripheral tissues, mainly including white adipose tissue and muscle.
The inhibited rate of $\mathrm{HGO}$ in AONW rats was reduced to $38 \%$ of that in CG rats, which demonstrated hepatic insulin resistance. These results indicate that hepatic insulin resistance was probably more serious than insulin resistance in peripheral tissues.

In the liver, AONW rats showed obvious lipidosis, mitochondrial swelling, and decreased mitochondrial cristae, while similar changes were not found by light and electronic microscopy in skeletal muscle. The more serious 


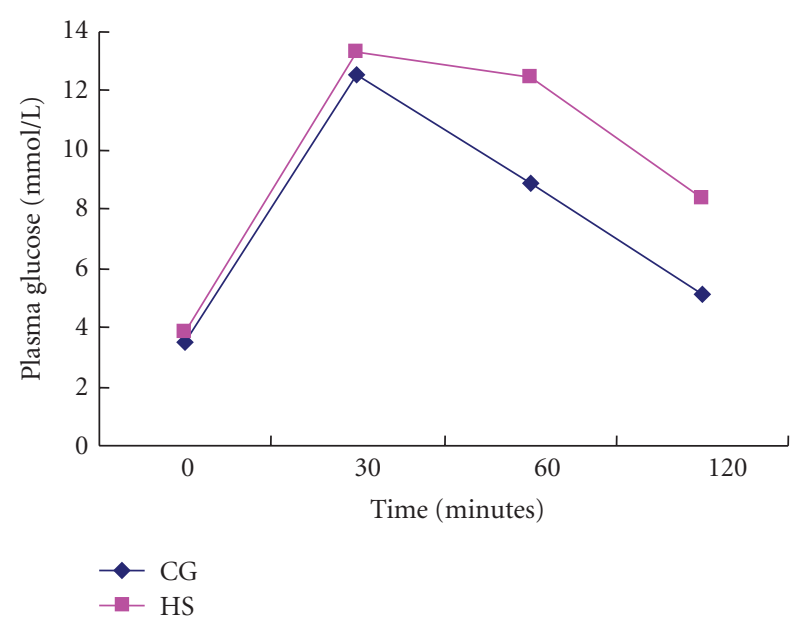

Figure 4: Plasma glucose (mmol/L) of rats fed CG food or HS food during intraperitoneal glucose tolerance tests. Values are means \pm $\mathrm{SD}, n=9, P=0.01$ for the difference between groups. CG: control group. HS: high-sucrose group.

morphologic changes in liver than in muscle were consistent with the findings from the hyperinsulinemic-euglycemic clamp. These findings imply that the hepatic insulin resistance developed earlier and was more serious than the insulin resistance in peripheral tissues (mainly muscle). These findings are also supported by other studies on rat obesity models that showed a tissue-specific sequence in the development of diet-induced insulin resistance $[15,16]$.

A few lipid droplets and capillary necrosis were observed in the muscle of AONW rats, while mitochondrial abnormalities and other morphologic changes were not observed. To detect whether there were gene modulations involved in FFA metabolism, mitochondrial function, and glucose uptake in the muscle without obvious morphologic changes, we measured mRNA expressions of UCP3, PGC- $1 \alpha$, TFAM, GLUT4, and IRS-1.

The UCP3 mRNA expression was significantly increased by high plasma FFA levels in the muscle of AONW rats, like in other situations involving elevated plasma FFA levels, including the fasting state, exercise, streptomycin-induced diabetes, and high-fat diets [17-19]. Because UCP3 has demonstrated the ability to increase fatty acid transport and oxidation [20] and protect mitochondria against lipidinduced mitochondrial dysfunction [21], increased UCP3 mRNA levels could be regarded as a marker of more FFA available to mitochondria or increased FFA metabolism. Other studies have shown that short-term elevated expression of UCP3 in transgenic rats protected rats from high-fat diet-induced insulin resistance $[22,23]$, while a continuous high-fat diet for 4 to 8 months eventually caused insulin resistance, although body weight increased less in controls [23]. Thus, as a skeletal muscle-specific UCP, the adaptively increased UCP3 mRNA might account for the decreased insulin resistance in muscles and relatively milder morphologic changes in muscular mitochondria and cells compared with those in the liver.

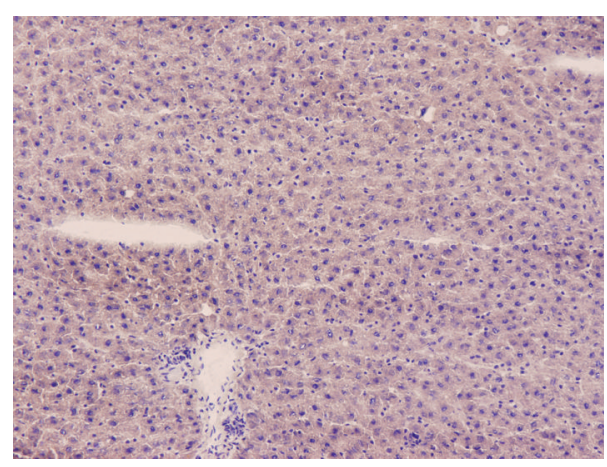

(a) CG

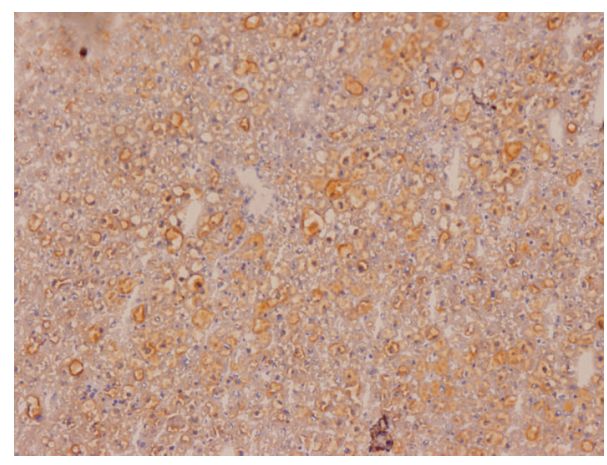

(b) HS

FIGURE 5: Liver in Sudan III stain under light microscopy, $\times 150$. (a) CG: normal. (b) HS: developed hepatic lipidosis. CG: control group. HS: high-sucrose group.

The present study showed increased expression of PGC$1 \alpha$ mRNA in rat muscle induced by an HS diet and a subsequent high plasma FFA level. However, PGC- $1 \alpha$ and some genes required for mitochondrial oxidative phosphorylation in skeletal muscle were downregulated with 3 days and 3 weeks of a high-fat diet in humans and mice, respectively [24]. Another study showed that acipimox-induced reduction in plasma FFA levels led to decreased expression of PGC- $1 \alpha$ in muscle of insulin-resistant individuals [25]. In these studies, PGC- $1 \alpha$ responded to changes in the plasma FFA level in a paradoxical manner. It is possible that the expression level of PGC- $1 \alpha$ caused by oversupply of FFA is dependent on the duration of exposure to FFA. Another possibility is that the composition of FFA causes different responses of PGC- $1 \alpha$ [26]. Recent studies have shown that PGC- $1 \alpha$ improves fatty acid oxidation and increases gene expression involved in mitochondrial biogenesis and function in cultured skeletal muscle cells $[27,28]$. These effects of PGC- $1 \alpha$ would be expected to lead to a reduction in lipid accumulation in muscle and an increase in insulin sensitivity [29]. Therefore, the increased PGC- $1 \alpha$ in this study might be a compensatory mechanism against fat deposition and insulin resistance in skeletal muscle, although eventual insulin resistance cannot be avoided.

TFAM mRNA expression did not change significantly in the muscle of AONW rats. As a key activator of mitochondrial transcription, TFAM stabilizes mitochondrial DNA 


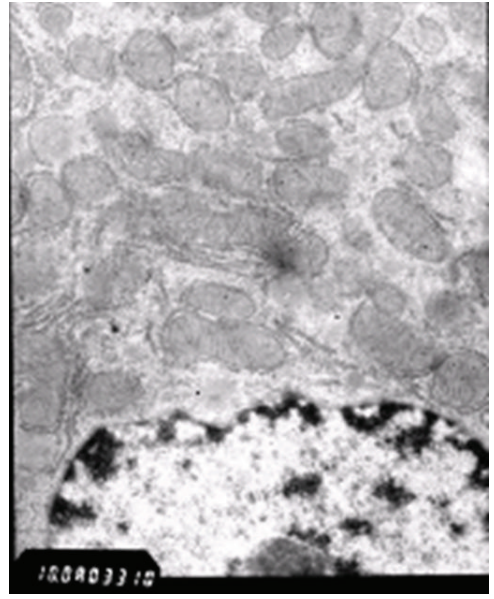

(a) CG

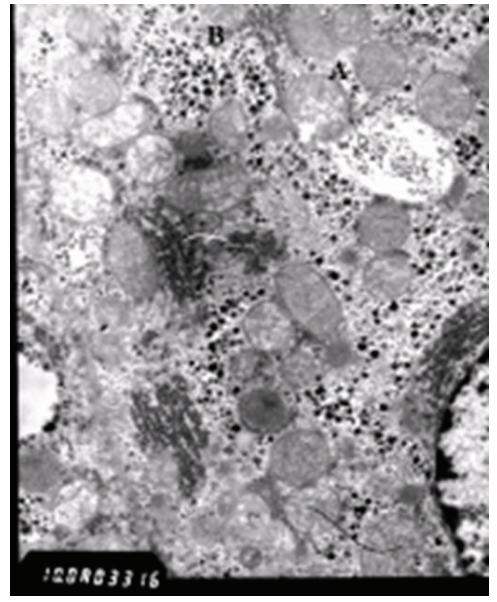

(b) HS

FIGURE 6: Liver under electronic microscopy. (a) CG: normal. (b) HS: mitochondrial swelling was obvious, and mitochondrial cristae were decreased (A) in the HS. More glycogenosomes were shown in the HS (B). CG: control group. HS: high-sucrose group.

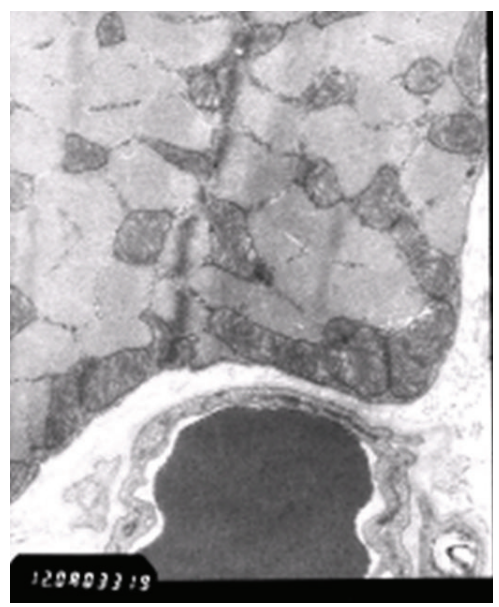

(a) CG

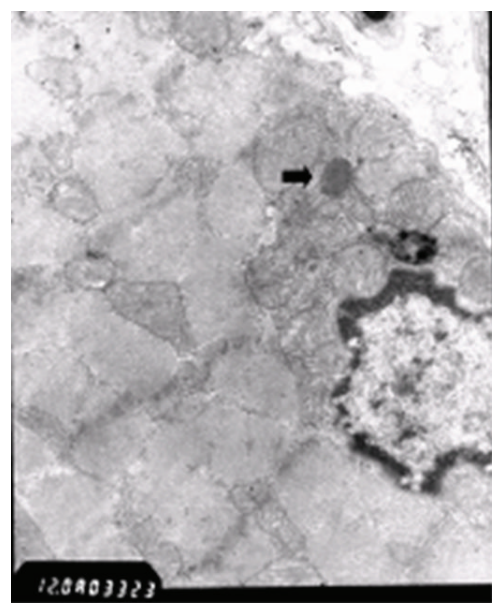

(b) HS

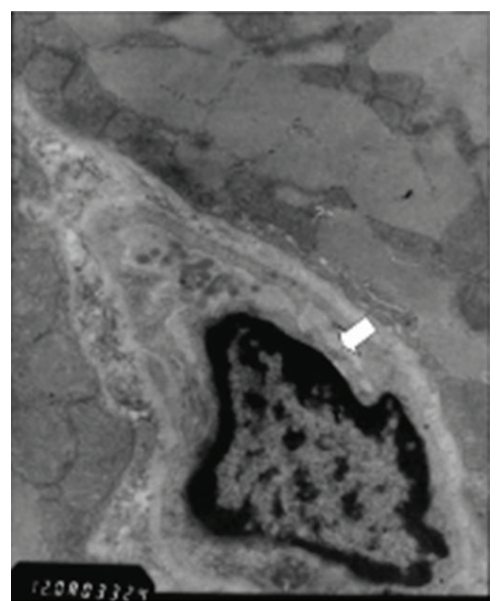

(c) HS

Figure 7: Muscle under electronic microscopy. (a, b) The morphology of mitochondria did not differ between groups. (b) Lipid droplets were found in HS muscle (black arrow). (c) Capillary necrosis was detected among muscle fibers of the HS (white arrow). CG: control group. HS: high-sucrose group.

through formation of the nucleoid and titrates the amount of mitochondrial DNA [30]. In this study, no obvious change in mitochondrial density was observed by microscopy, although mitochondrial counting was not performed. The morphologic findings of the mitochondria were consistent with the TFAM mRNA expression. As in another study [24], TFAM mRNA did not change consistently with PGC1- $\alpha$ in the present study, which implies the presence of an unrecognized regulation pathway.

No significant changes in IRS-1 or GLUT4 mRNA expression were found in AONW rats. Whether there was dysfunction in tyrosine phosphorylation of IRS-1 and GLUT4 transposition was not determined in this study [31, 32].
Whether IRS-1 and GLUT4 were involved in the insulin resistance of this AONW rat model was also not determined.

There are limitation of this study. First, genes involved in fatty acid metabolism and glucose uptake were not detected. Second, mitochondrial content was not measured and genes involved in mitochondrial biogenesis were detected.

In summary, the present study showed an AONW rat model developed by a modified HS diet. The AONW model mimics some metabolic features of MONW individuals, such as increased visceral adipose, high plasma FFA, and insulin resistance. Morphologic impairment and insulin resistance of the liver were more serious than those in the skeletal muscle in this model. In the less impaired muscle, increases 


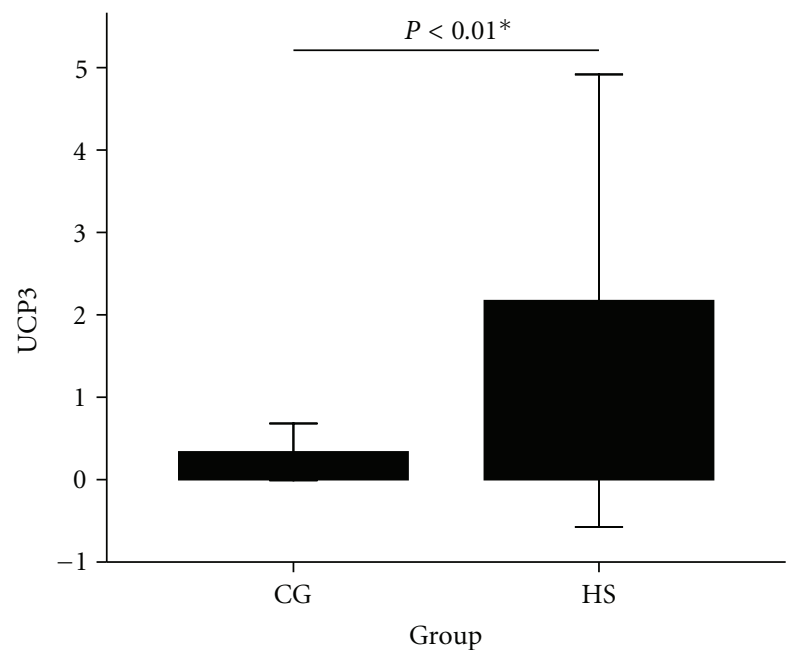

(a)

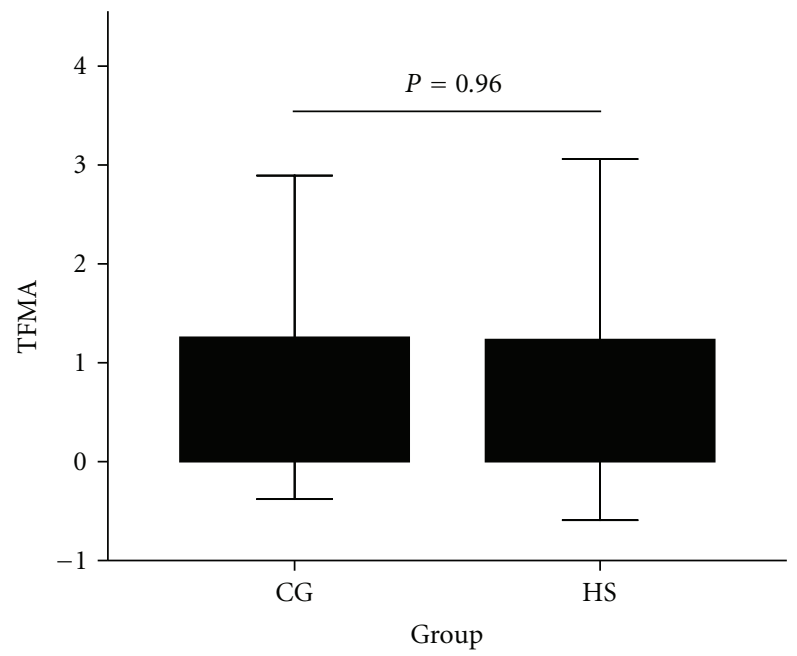

(c)

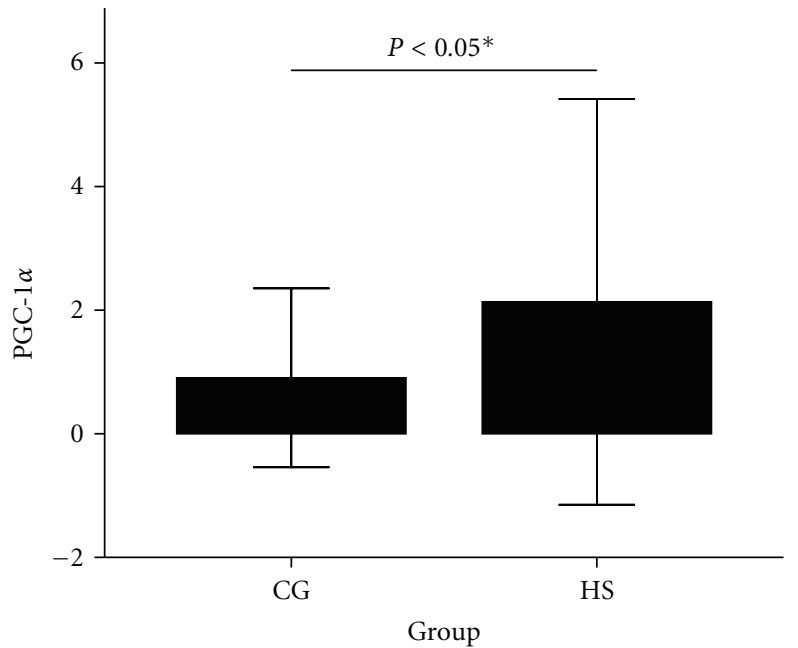

(b)

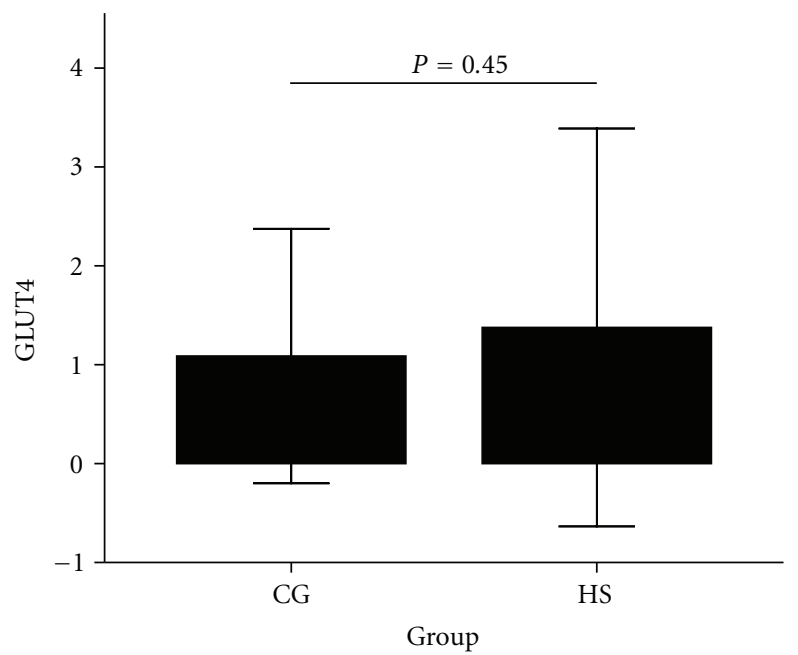

(d)

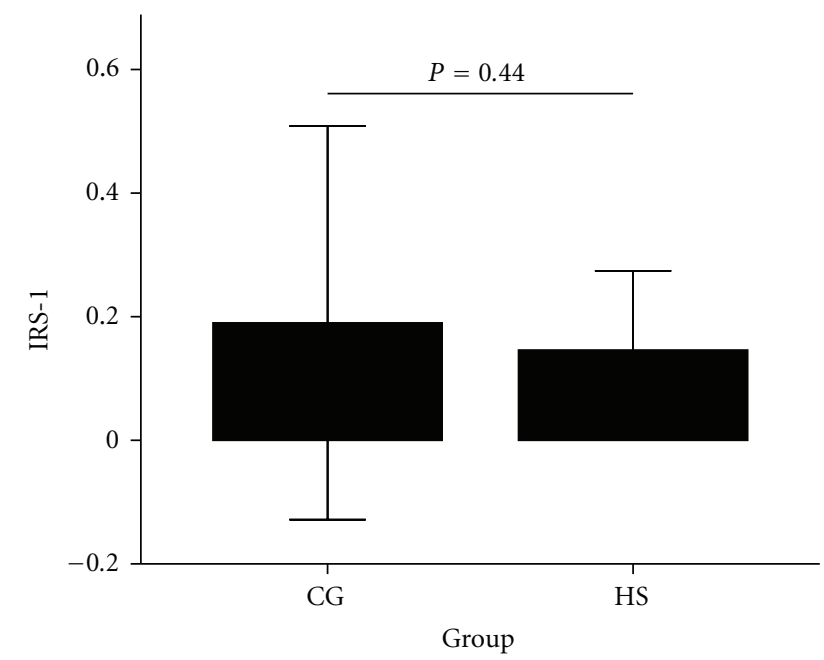

(e)

FIGURE 8: mRNA expression for genes involved in mitochondrial oxidation and biogenesis. (a) UCP3, (b) PGC-1 $\alpha$, (c) TFAM, (d) GLUT4, and (e) IRS-1. The error bar is \pm 2 SD. ${ }^{*} P<0.05,{ }^{* *} P<0.01$, HS versus CG. CG: control group. HS: high-sucrose group. 
in UCP3 and PGC-1 $\alpha$ mRNA expression are probably responding to increased available FFA and might protect muscle from insulin resistance to some extent.

\section{Authors' Contribution}

L. Cao, X. Liu, and H. Cao contributed equally in the work of this paper.

\section{References}

[1] N. B. Ruderman, S. H. Schneider, and P. Berchtold, "The "metabolically-obese," normal-weight individual," American Journal of Clinical Nutrition, vol. 34, no. 8, pp. 1617-1621, 1981.

[2] E. Molero-Conejo, L. M. Morales, V. Fernández et al., "Lean adolescents with increased risk for metabolic syndrome," Archivos Latinoamericanos de Nutricion, vol. 53, no. 1, pp. 3946, 2003.

[3] R. V. Dvorak, W. F. DeNino, P. A. Ades, and E. T. Poehlman, "Phenotypic characteristics associated with insulin resistance in metabolically obese but normal-weight young women," Diabetes, vol. 48, no. 11, pp. 2210-2214, 1999.

[4] M. P. St-Onge, I. Janssen, and S. B. Heymsfield, "Metabolic syndrome in normal-weight Americans: new definition of the metabolically obese, normal-weight individual," Diabetes Care, vol. 27, no. 9, pp. 2222-2228, 2004.

[5] F. Conus, D. B. Allison, R. Rabasa-Lhoret et al., "Metabolic and behavioral characteristics of metabolically obese but normal-weight women," Journal of Clinical Endocrinology and Metabolism, vol. 89, no. 10, pp. 5013-5020, 2004.

[6] Y. W. Park, S. Zhu, L. Palaniappan, S. Heshka, M. R. Carnethon, and S. B. Heymsfield, "The metabolic syndrome: prevalence and associated risk factor findings in the US population from the Third National Health and Nutrition Examination Survey, 1988-1994," Archives of Internal Medicine, vol. 163, no. 4, pp. 427-436, 2003.

[7] N. Ruderman, D. Chisholm, X. Pi-Sunyer, and S. Schneider, "The metabolically obese, normal-weight individual revisited," Diabetes, vol. 47, no. 5, pp. 699-713, 1998.

[8] A. Katsuki, Y. Sumida, H. Urakawa et al., "Increased visceral fat and serum levels of triglyceride are associated with insulin resistance in Japanese metabolically obese, normal weight subjects with normal glucose tolerance," Diabetes Care, vol. 26, no. 8, pp. 2341-2344, 2003.

[9] M. P. Cleary, J. R. Vasselli, and M. R. Greenwood, "Development of obesity in Zucker obese (fafa) rat in absence of hyperphagia," The American Journal of Physiology, vol. 238, no. 3, pp. E284-E292, 1980.

[10] D. N. Brindley and J. C. Russell, "Animal models of insulin resistance and cardiovascular disease: some therapeutic approaches using the JCR:LA-cp rat," Diabetes, Obesity and Metabolism, vol. 4, no. 1, pp. 1-10, 2002.

[11] J. Sassard, A. Larcan, J. D. Sraer et al., "Human essential hypertension and genetic hypertension in rats: The Lyon model," Bulletin de l'Academie Nationale de Medecine, vol. 190, no. 1, pp. 111-121, 2006.

[12] L. Li, G. Yang, Q. Li, Y. Tang, and K. Li, "High-fat- and lipidinduced insulin resistance in rats: The comparison of glucose metabolism, plasma resistin and adiponectin levels," Annals of Nutrition and Metabolism, vol. 50, no. 6, pp. 499-505, 2007.
[13] S. Tanaka, T. Hayashi, T. Toyoda et al., "High-fat diet impairs the effects of a single bout of endurance exercise on glucose transport and insulin sensitivity in rat skeletal muscle," Metabolism, vol. 56, no. 12, pp. 1719-1728, 2007.

[14] C. Raffaella, B. Francesca, F. Italia, P. Marina, L. Giovanna, and I. Susanna, "Alterations in hepatic mitochondrial compartment in a model of obesity and insulin resistance," Obesity, vol. 16, no. 5, pp. 958-964, 2008.

[15] R. Kleemann, M. Van Erk, L. Verschuren et al., "Time-resolved and tissue-specific systems analysis of the pathogenesis of insulin resistance," PLoS ONE, vol. 5, no. 1, article e8817, 2010.

[16] M. J. Pagliassotti and P. A. Prach, "Quantity of sucrose alters the tissue pattern and time course of insulin resistance in young rats," American Journal of Physiology, vol. 269, no. 3, part 2, pp. R641-R646, 1995.

[17] M. P. Thompson and D. Kim, "Links between fatty acids and expression of UCP2 and UCP3 mRNAs," FEBS Letters, vol. 568, no. 1-3, pp. 4-9, 2004.

[18] J. Matsuda, K. Hosoda, H. Itoh et al., "Cloning of rat uncoupling protein-3 and uncoupling protein-2 cDNAs: their gene expression in rats fed high-fat diet," FEBS Letters, vol. 418, no. 1-2, pp. 200-204, 1997.

[19] D. W. Gong, Y. He, and M. L. Reitman, "Genomic organization and regulation by dietary fat of the uncoupling protein 3 and 2 genes," Biochemical and Biophysical Research Communications, vol. 256, no. 1, pp. 27-32, 1999.

[20] V. Bezaire, L. L. Spriet, S. Campbell et al., "Constitutive UCP3 overexpression at physiological levels increases mouse skeletal muscle capacity for fatty acid transport and oxidation," FASEB Journal, vol. 19, no. 8, pp. 977-979, 2005.

[21] M. Nabben, J. Hoeks, E. Moonen-Kornips et al., "Significance of uncoupling protein 3 in mitochondrial function upon midand long-term dietary high-fat exposure," FEBS Letters, vol. 585, no. 24, pp. 4010-4017, 2011.

[22] C. S. Choi, J. J. Fillmore, J. K. Kim et al., "Overexpression of uncoupling protein 3 in skeletal muscle protects against fatinduced insulin resistance," Journal of Clinical Investigation, vol. 117, no. 7, pp. 1995-2003, 2007.

[23] S. R. Costford, S. N. Chaudhry, S. A. Crawford, M. Salkhordeh, and M. E. Harper, "Long-term high-fat feeding induces greater fat storage in mice lacking UCP3," American Journal of Physiology, vol. 295, no. 5, pp. E1018-E1024, 2008.

[24] L. M. Sparks, H. Xie, R. A. Koza et al., "A high-fat diet coordinately downregulates genes required for mitochondrial oxidative phosphorylation in skeletal muscle," Diabetes, vol. 54, no. 7, pp. 1926-1933, 2005.

[25] M. Bajaj, R. Medina-Navarro, S. Suraamornkul, C. Meyer, R. A. DeFronzo, and L. J. Mandarino, "Paradoxical changes in muscle gene expression in insulin-resistant subjects after sustained reduction in plasma free fatty acid concentration," Diabetes, vol. 56, no. 3, pp. 743-752, 2007.

[26] H. Staiger, K. Staiger, C. Haas, M. Weisser, F. MacHicao, and H. U. Häring, "Fatty acid-induced differential regulation of the genes encoding peroxisome proliferator-activated receptor- $\gamma$ coactivator- $1 \alpha$ and $-1 \beta$ in human skeletal muscle cells that have been differentiated in vitro," Diabetologia, vol. 48 , no. 10 , pp. 2115-2118, 2005.

[27] E. Mormeneo, C. Jimenez-Mallebrera, X. Palomer et al., "PGC-1alpha induces mitochondrial and myokine transcriptional programs and lipid droplet and glycogen accumulation in cultured human skeletal muscle cells," PLoS One, vol. 7, no. 1, article e29985, 2012.

[28] N. Nikolic, M. Rhedin, A. C. Rustan et al., "Overexpression of PGC-1alpha increases fatty acid oxidative capacity of human 
skeletal muscle cells," Biochemistry Research International, vol. 2012, Article ID 714074, 2012.

[29] C. R. Benton, J. G. Nickerson, J. Lally et al., "Modest PGC$1 \alpha$ overexpression in muscle in vivo is sufficient to increase insulin sensitivity and palmitate oxidation in subsarcolemmal, not intermyofibrillar, mitochondria," Journal of Biological Chemistry, vol. 283, no. 7, pp. 4228-4240, 2008.

[30] D. Kang, S. H. Kim, and N. Hamasaki, "Mitochondrial transcription factor A (TFAM): roles in maintenance of mtDNA and cellular functions," Mitochondrion, vol. 7, no. 1-2, pp. 3944, 2007.

[31] R. M. N. Bezerra, M. Ueno, M. S. Silva, D. Q. Tavares, C. R. O. Carvalho, and M. J. A. Saad, "A high fructose diet affects the early steps of insulin action in muscle and liver of rats," Journal of Nutrition, vol. 130, no. 6, pp. 1531-1535, 2000.

[32] P. A. Hansen, D. H. Han, B. A. Marshall et al., "A high fat diet impairs stimulation of glucose transport in muscle: functional evaluation of potential mechanisms," Journal of Biological Chemistry, vol. 273, no. 40, pp. 26157-26163, 1998. 


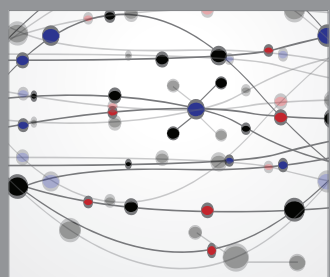

The Scientific World Journal
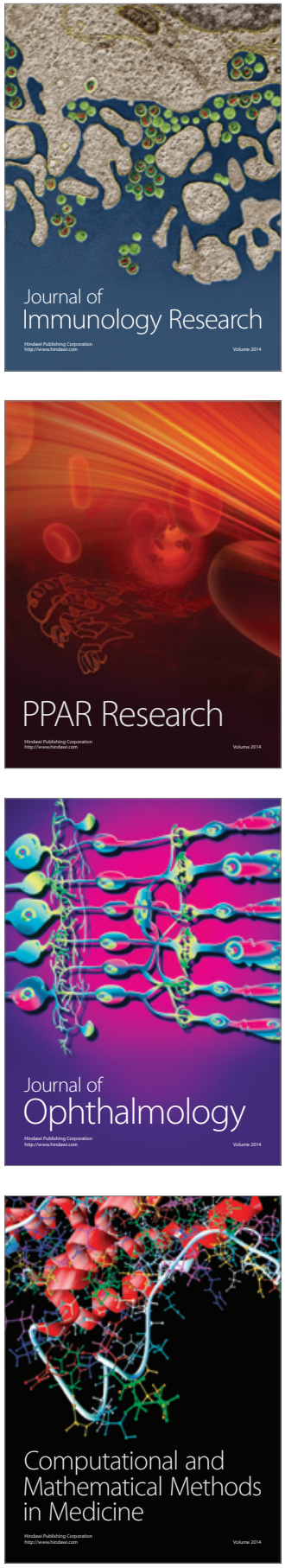

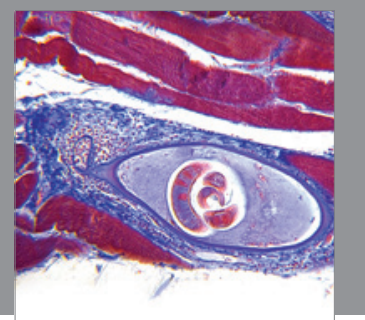

Gastroenterology

Research and Practice
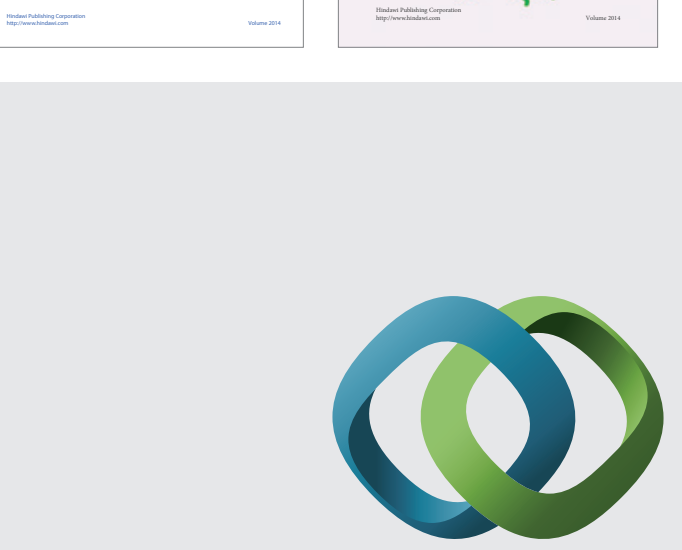

\section{Hindawi}

Submit your manuscripts at

http://www.hindawi.com
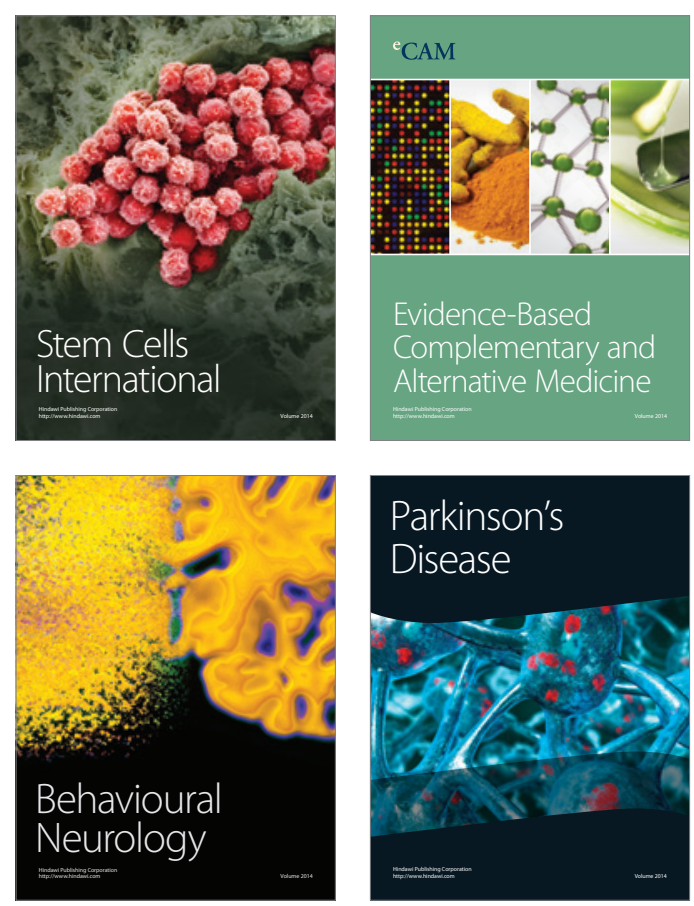

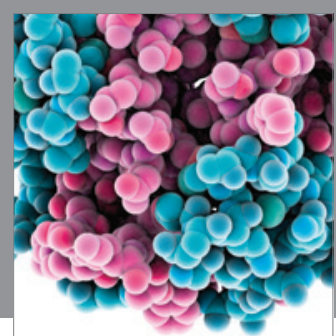

Journal of
Diabetes Research

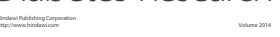

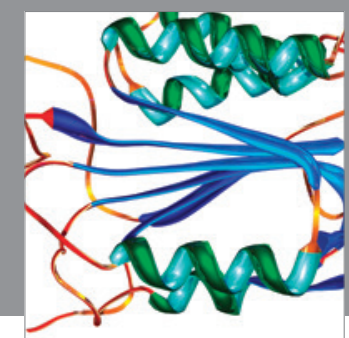

Disease Markers
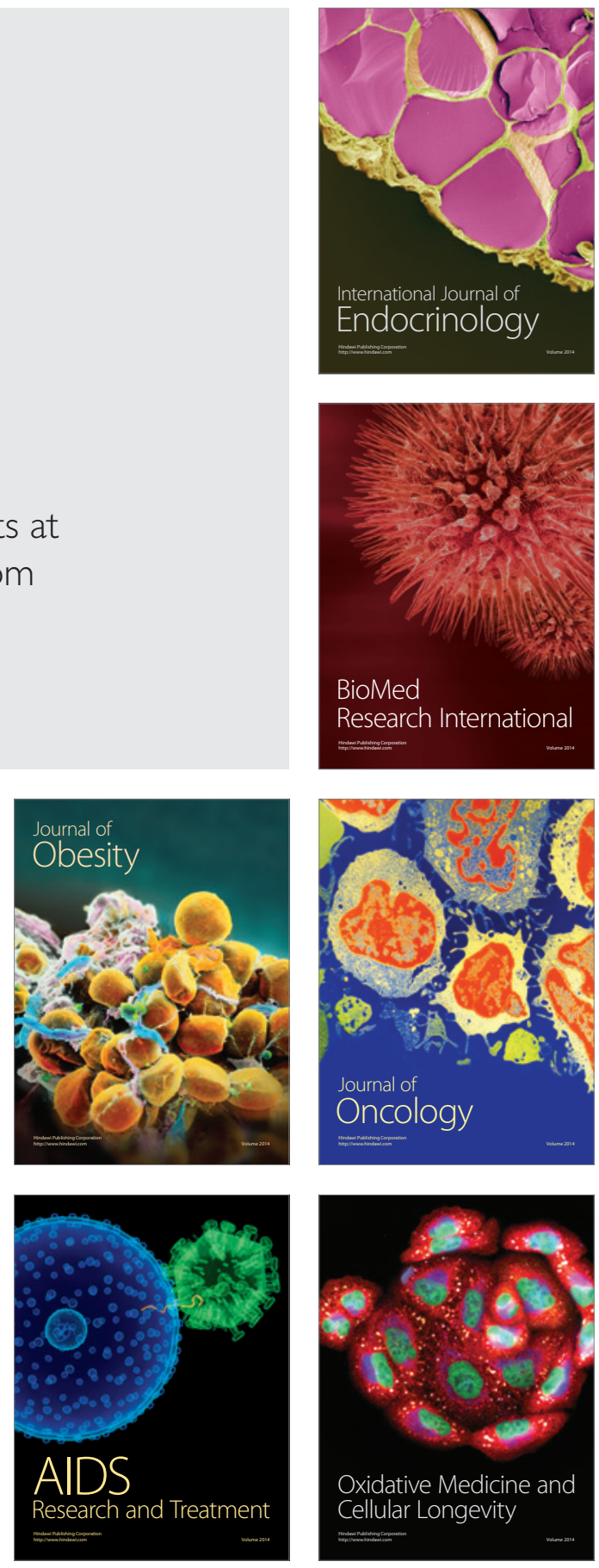